\title{
White Matter Correlates of Cognitive Impairment in Essential Tremor
}

\author{
K.S. Bhalsing, K.J. Kumar, J. Saini, R. Yadav, A.K. Gupta, and P.K. Pal
} 厓

\begin{abstract}
BACKGROUND AND PURPOSE: Impairment of cognitive functions occurs in essential tremor, though the mechanism is largely unknown. The aim of this study was to find microstructural correlates of cognitive dysfunction seen in essential tremor by using DTI and neuropsychological assessment.
\end{abstract}

MATERIALS AND METHODS: Fifty-five patients with essential tremor and 55 matched healthy controls were evaluated. Essential tremor was diagnosed by using the National Institutes of Health criteria. Subjects were assessed by using a structured neuropsychological battery. DTI data were acquired by using 3T MR imaging and were analyzed by using tract-based spatial statistics. Fractional anisotropy, mean diffusivity, radial diffusivity, and axial diffusivity were analyzed.

RESULTS: Patients were considered cognitively impaired when the test score was 1.5 SDs below or above the mean of healthy controls (depending on the type of test) in $\geq 3$ neuropsychological tests. Patients with cognitive impairment had significantly higher mean diffusivity, radial diffusivity, and axial diffusivity values in the bilateral frontoparietal regions. In patients with cognitive impairment, mean diffusivity, radial diffusivity, and axial diffusivity showed correlations with various neuropsychological test scores. Executive function correlated with DTI measures of the frontal white matter, cingulum, inferior superior longitudinal and uncinate fasciculi, anterior thalamic radiations, and posterior lobe of the cerebellum. Visuospatial function correlated with the right parieto-occipital lobe, whereas visualverbal memories correlated with the anterior thalamic radiations, inferior longitudinal and uncinate fasciculi, and the posterior lobe of the cerebellum. No significant correlations were found between fractional anisotropy and any of the neuropsychological test scores.

CONCLUSIONS: The present study demonstrates a correlation between neuropsychological test scores and DTI measures, suggesting a neuroanatomic basis for cognitive impairment seen in patients with essential tremor.

ABBREVIATIONS: $\mathrm{ET}=$ essential tremor; $\mathrm{ETCI}=\mathrm{ET}$ with cognitive impairment; $\mathrm{ETNCI}=\mathrm{ET}$ without cognitive impairment; $\mathrm{HC}=$ healthy controls; $\mathrm{TBSS}=$ tract-based spatial statistics

$E$ sential tremor (ET) is the most common movement disorder. -It is associated with cognitive impairments ${ }^{1}$ and behavioral and sleep disturbances, ${ }^{2}$ in addition to tremor. Various studies have shown cognitive impairment, especially executive-visuospatial dysfunction and attention deficits, in patients with ET. ${ }^{3,4}$ Voxel-based morphometry studies in ET have found volume reductions in the cerebellum and cerebrum (frontal lobe, insula, and cingulum). ${ }^{5,6}$ While voxel-based morphometry analysis is

Received May 9, 2014; accepted after revision August 22.

From the Departments of Neurology (K.S.B., R.Y., P.K.P.), Clinical Psychology (K.J.K.), and Neuroimaging and Interventional Radiology (J.S., A.K.G.), National Institute of Mental Health and Neurosciences, Karnataka, India.

Please address correspondence to Pramod Kumar Pal, DM, Department of Neurology, National Institute of Mental Health and Neurosciences, Hosur Rd, Bangalore560029, Karnataka, India; e-mail: pal.pramod@rediffmail.com

Indicates article with supplemental on-line tables.

Evidence-Based Medicine Level 2.

http://dx.doi.org/10.3174/ajnr.A4138 useful for demonstrating regional GM changes, DTI provides information about the microstructural organization of WM. The most widely used measurements are fractional anisotropy and mean diffusivity. Variations in these measures within WM structures are thought to reflect structural changes such as myelin abnormalities, neuronal damage, microtubule breakdown, and/or axonal loss. The involvement of WM is controversial in ET. In particular, the first diffusion study in ET did not report WM abnormalities, ${ }^{7}$ while Shin et $\mathrm{al}^{8}$ showed fractional anisotropy reduction in the cerebellum, brain stem, and cerebral hemispheres in patients with ET. Recently, a few studies have reported the presence of WM abnormalities in the frontoparietal region and cerebellar peduncles. ${ }^{9,10}$ Thus, structural studies in ET suggest involvement of the cerebellofrontal network, which is known to play an important role in higher level cognitive functions. ${ }^{11}$

The aim of the present study was to characterize the brain localization of cognitive dysfunction in ET. To characterize cognitive dysfunction, we used a structured neuropsychological bat- 
tery. Tract-based spatial statistics (TBSS; http://fsl.fmrib.ox. ac.uk/fsl/fslwiki/TBSS) analysis on DTI images was performed to characterize the microstructural correlates of cognitive performance. Such correlation analysis offers an opportunity to detect the role of WM microstructural damage in cognitive deficits seen in ET.

\section{MATERIALS AND METHODS}

We studied 55 patients with ET (mean age, $45.6 \pm 11.7$ years) and 55 age-, sex-, education-, and handedness-matched healthy controls (HC) (mean age, $46.2 \pm 11.4$ years) at the National Institute of Mental Health and Neurosciences, India, from December 2011 to August 2013. All subjects were screened with the Mini-Mental State Examination scale and were included if the score was $\geq 25$.

\section{Patient Group}

All patients recruited in our study were carefully examined by a movement disorder specialist (P.K.P.). The diagnosis of ET was confirmed by using the National Institutes of Health Collaborative Genetic Criteria. ${ }^{12}$ The severity of the tremor was assessed by using the Fahn-Tolosa-Marin tremor rating scale. In all patients, arm tremors were assessed at rest, with their arms extended, and while performing finger-to-nose movements. Head, voice, leg, and trunk tremors were also assessed in addition to arm tremor. Tremor severity was also assessed while writing and performing activities of daily living (such as pouring water and buttoning and unbuttoning a shirt).

The half-life of the commonly used medications for tremor ranges from 5 to 50 hours, and it takes 3-5 plasma half-lives to eliminate a drug from a body. Therefore, patients who were on medications for tremor were asked to stop the medications 2 weeks before the study.

\section{Control Group}

Hospital staff, spouses, and nonfamily attendants of patients were recruited as controls. Each control was carefully interviewed and examined to rule out any neurologic conditions and family history of neurodegenerative disorders. The Modified Mini Screen was applied to all of them to rule out the presence of any psychiatric disorder.

The study was approved by the ethics committee of the institution, and all subjects consented to participate in this study.

\section{Neuropsychological Evaluation}

All subjects underwent neuropsychological assessment with tests assessing attention, executive functions, memory (visual, verbal, and working memory), and visuospatial functions. In patients, depending on the type of test, a test finding was considered abnormal if the score was 1.5 SDs below or above the mean of the healthy controls. ${ }^{13}$ A patient was recognized as cognitively impaired if $\geq 3$ neuropsychological test findings were abnormal. We considered $\geq 3$ tests to increase the stringency of the criteria. Patients were further divided into 2 groups, namely ET with cognitive impairment (ETCI) and ET without cognitive impairment (ETNCI).

\section{Test for Attention and Executive Functions}

Wisconsin Card Sorting Test. A computerized version of the Wisconsin Card Sorting Test (Berg's Card Sorting Test; http:// pebl.sourceforge.net/battery.html), which consists of 64 trials, was used. Performance was evaluated on the basis of categories completed, perseverative response, and perseverative errors.

Stroop Test. The Stroop Test is a test of response inhibition. It consists of 3 subtasks having 100 stimuli each. The first subtask shows names of colors printed in black ink in random order. The second subtask displays 1 of 4 basic colors. The third subtask contains names of colors printed in an incongruous ink color; for example, the word "yellow" printed in red ink. The subject is required to inhibit the prepotent or habitual response of reading the word and instead give a new response, which is to name the color of the ink. This is known as Stroop effect.

The time needed to complete each subtask was recorded. Performance was evaluated on the basis of "interference" because it is considered a measure of cognitive flexibility and executive functioning. ${ }^{14}$ It was calculated by subtracting the average time needed to complete the first 2 subtasks from the time needed to complete the third subtask.

Color Trails Tests I and II. The Color Trails Test has 3 parts. The time required to perform each part was recorded.

\section{Test for Visuospatial Functions}

Line Orientation Test. The Benton's Judgment of Line Orientation Test is considered a valid task for the assessment of visuospatial function. ${ }^{15}$ In the present study, we used a self-designed short form of the test (15 items instead of 30), keeping all the parameters similar to those in the original test.

\section{Test for Working Memory}

Wechsler Memory Scale III Spatial Span Test. We performed both forward and backward Spatial Span tests to judge the performance.

\section{Test for Verbal and Visual Memory}

We used the Rey Auditory Verbal Learning Test and Wechsler Memory Scale III Face Recognition test for evaluation of verbal and visual memories, respectively. In both tests, performance was evaluated on the basis of immediate and delayed recall.

\section{MR Imaging Acquisition}

Imaging was performed in all subjects by using an Achieva (Philips Healthcare, Best, the Netherlands) 3T MR imaging scanner. Diffusion-weighted data with 16 directions were acquired by using a 32-channel head coil. In case of severe head tremors, additional cushions were used to stabilize the head. The acquisition parameters were the following: $\mathrm{TR} / \mathrm{TE}=8782 / 62 \mathrm{~ms}$, flip angle $=$ $90^{\circ}, b=1000 \mathrm{~s} / \mathrm{mm}^{2}$, section thickness $=2 \mathrm{~mm}$, number of sections $=60$, intersection gap $=0$, acquisition matrix $=112 \times 112$. FLAIR images were also acquired. Before the analysis, all images were screened by a neuroradiologist for the presence of structural abnormalities.

\section{Tract-Based Spatial Statistics}

The fMRI of the Brain Software Library (FSL; http://www.fmrib. ox.ac.uk/fsl), Tract-Based Spatial Statistics tool, ${ }^{16}$ was used to 
Clinical and demographic details of study subjects

\begin{tabular}{lcccc}
\hline & $\mathrm{ETCl}(\boldsymbol{n}=\mathbf{3 3})$ & $\mathrm{ETNCI}(\boldsymbol{n}=\mathbf{2 2})$ & $\mathrm{HC}(\boldsymbol{n}=\mathbf{5 5})$ & $\boldsymbol{P}$ Value \\
\hline Age (yr) & $47.03 \pm 10.4$ & $43.4 \pm 13.4$ & $46 \pm 11$ & $.2^{\mathrm{a}}$ \\
Sex (female/male) & $1: 2.8$ & $1: 2.5$ & $1: 2.6$ & $.4^{\mathrm{a}}$ \\
MMSE score & $29.2 \pm 0.86$ & $29.6 \pm 0.7$ & $29.9 \pm 0.2$ & $.1^{\mathrm{a}}$ \\
Family history of disease (No.) & 18 & 13 & $\mathrm{NA}$ & $.6^{\mathrm{b}}$ \\
Disease duration (yr) & $10.2 \pm 7.4$ & $9.5 \pm 8.2$ & $\mathrm{NA}$ & $.8^{\mathrm{b}}$ \\
Fahn-Tolosa-Marin score & $34.8 \pm 14.3$ & $33.9 \pm 15.4$ & $\mathrm{NA}$ & $.8^{\mathrm{b}}$ \\
\hline
\end{tabular}

Note:-NA indicates not applicable; MMSE, Mini-Mental State Examination.

${ }^{a}$ ANOVA.

${ }^{\mathrm{b}}$ Unpaired $t$ test.

compare diffusion measures among ETCI, ETNCI, and HC groups and to correlate diffusion measures with neuropsychological assessment scores. DTI data were first corrected for eddy current and head motion. Fractional anisotropy images were then created by fitting a tensor model to the raw diffusion data by using the FMRIB Diffusion Toolbox (http://www.fmrib.ox.ac.uk/fsl/ fdt/index.html) and were extracted by using the Brain Extraction Tool. ${ }^{17,18}$ The fractional anisotropy data of all the subjects were then aligned into a common space by using the FMRIB Nonlinear Registration Tool (FNIRT; http://fsl.fmrib.ox.ac.uk/fsl/fslwiki/ FNIRT). In the next step, the mean fractional anisotropy image was created and thinned to create a mean fractional anisotropy skeleton, which represented the centers of all tracts common to the whole group. To exclude GM or cerebrospinal fluid, the mean fractional anisotropy skeleton was thresholded at 0.2 . The aligned fractional anisotropy data of each subject were then projected onto this fractional anisotropy skeleton. In addition to fractional anisotropy, mean diffusivity, radial diffusivity, and axial diffusivity images were also generated.

\section{Statistical Analysis}

F-test and Post Hoc Analysis. The F-test was performed among the 3 groups (ETCI, ETNCI, and HC) to investigate differences in DTI measures within the groups. Post hoc analysis was then performed (when appropriate) to evaluate the differences among ETCI versus HC, ETCI versus ETNCI, and ETNCI versus HC. Age, sex, total intracranial volume, disease duration, and FahnTolosa-Marin scale scores were entered as nuisance regressors in the design matrix throughout the analysis. Statistical analysis was performed by using the FSL Randomize Tool (http://fsl.fmrib.ox.ac.uk/ fsl/fslwiki/Randomise/UserGuide) with 5000 permutations.

Correlation Analysis. Correlation analysis was performed to study the relationship between neuropsychological test scores and each of the DTI measures. Neuropsychological test scores were used as a covariate of interest in the framework of a general linear model. Age, sex, total intracranial volume, disease duration, and Fahn-Tolosa-Marin scale scores were entered as nuisance regressors in the design matrix throughout the analysis. Statistical analysis was performed by using the FSL Randomize Tool with 5000 permutations.

Threshold-free cluster enhancement was performed to enhance cluster-like structures. ${ }^{19}$ Family-wise error correction with $P<.05$ was considered significant. In correlation analysis, we did not find any surviving clusters or voxels with analysis corrected for multiple comparisons (family-wise error or false discovery rate correction), so we decided to report the nal fasciculus. correlation results with $P<.001$ (uncorrected for multiple comparisons) as statistically significant.

\section{RESULTS}

Thirty-three patients were classified as ETCI. A comparison of the 3 groups with respect to demographic and clinical characteristics is presented in the Table. Intergroup analysis among ETCI, ETNCI, and HC by using ANOVA and between ETCI and ETNCI by using a $t$ test did not show any statistically significant differences in any of the demographic and clinical parameters.

\section{ANOVA and Post Hoc T Test}

An F-test performed among the 3 groups was found to be statistically significant in mean diffusivity, radial diffusivity, and axial diffusivity, while it was not significant for fractional anisotropy. On post hoc $t$ test analysis (family-wise error-corrected, $P<.05$ ), the ETCI group compared with HC group showed increased mean diffusivity in the right cingulum and left precuneus (parietal lobe), increased radial diffusivity in the right medial frontal white matter and left cingulum, and increased axial diffusivity in the right cingulum and left medial frontal white matter. The Figure shows the results of $t$ test analysis performed in ETCI versus HC.

There was no statistically significant difference in any of the diffusion measures for comparisons between ETCI versus ETNCI and ETNCI versus HC groups.

\section{Correlation Analysis}

The following correlations (uncorrected for multiple comparisons, $P<.001$ ) between diffusion measures and neuropsychology assessment scores were observed in the ETCI group (On-line Tables 1-3):

1) The number of categories completed on the Wisconsin Card Sorting Test showed a negative correlation with mean diffusivity, radial diffusivity, and axial diffusivity of the right medial frontal gyrus, right cingulum, left anterior thalamic radiations, and bilateral cerebellum (posterior lobe), whereas the number of perseverative errors on the Wisconsin Card Sorting Test positively correlated with mean diffusivity of bilateral anterior thalamic radiations, bilateral superior longitudinal fasciculus, left uncinate fasciculus, left cerebellum (posterior lobe) and radial diffusivity of left anterior thalamic radiations, right superior and inferior longitudinal fasciculus and left uncinate fasciculus.

2) The Stroop Test score showed a positive correlation with mean diffusivity, radial diffusivity, and axial diffusivity of the left anterior thalamic radiations, bilateral superior longitudinal fasciculus, left uncinate fasciculus, and left cerebellum (posterior lobe).

3) The Color Trails Test score positively correlated with mean diffusivity, radial diffusivity, and axial diffusivity of the left anterior thalamic radiations and bilateral superior longitudi-

4) Working memory scores showed a negative correlation with 

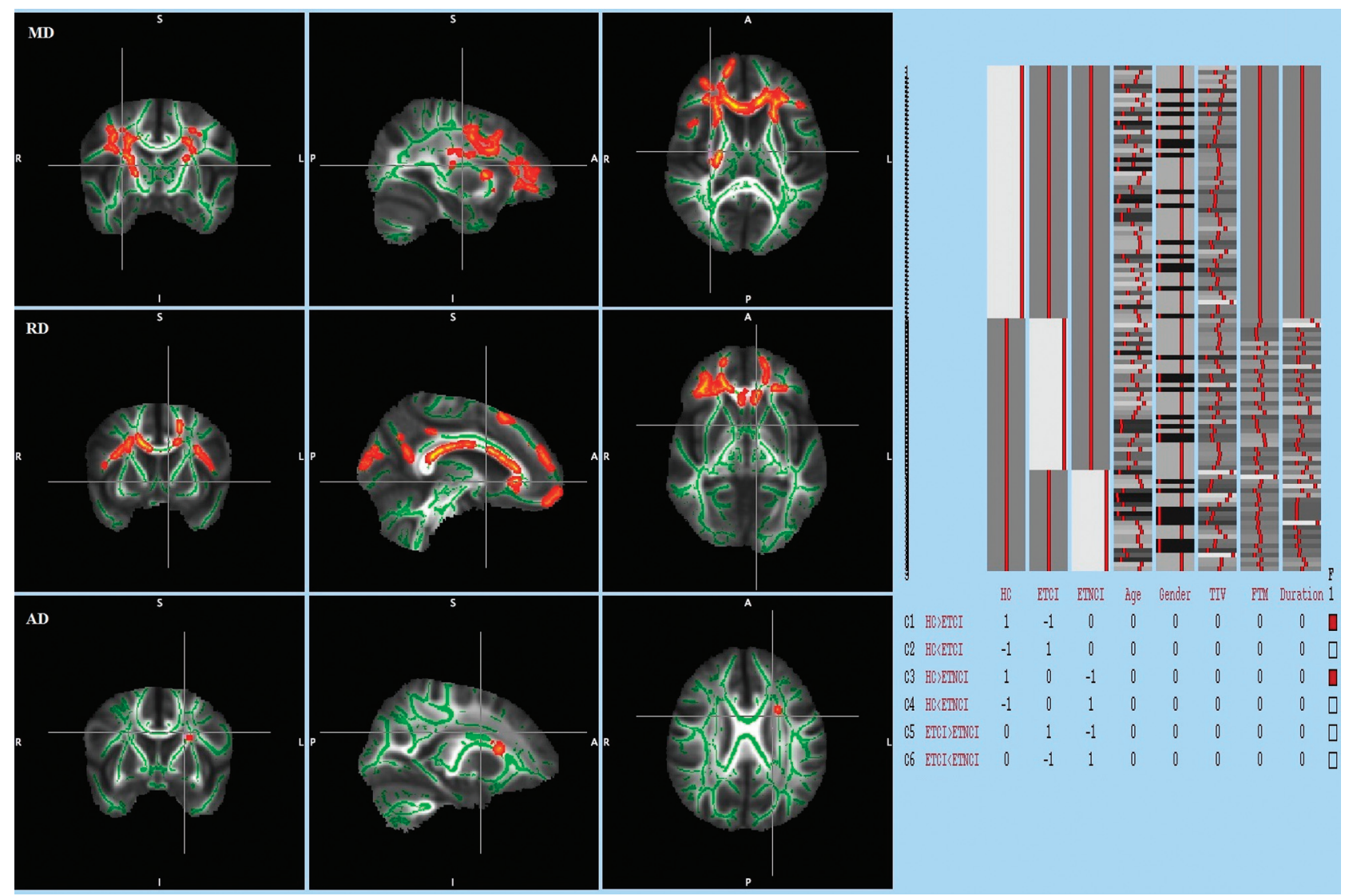

FIGURE. TBSS analysis ( $t$ test) performed in those with ETCI versus HC. Voxels demonstrating significantly (family-wise error-corrected $P<.05$ ) increased mean diffusivity, radial diffusivity, and axial diffusivity in $\mathrm{ETCl}$ compared with $\mathrm{HC}$ are shown in red-yellow. Voxels are thickened into local tracts and overlaid on the white matter skeleton (green). The design matrix used for the TBSS analysis is shown on the right.

mean diffusivity and radial diffusivity of the right medial frontal gyrus, right cingulum, bilateral anterior thalamic radiations, right superior longitudinal fasciculus, and right cerebellum (posterior lobe).

5) Visuospatial scores negatively correlated with mean diffusivity, radial diffusivity of the right parietal lobe (supramarginal), and the right occipital lobe.

6) Verbal memory immediate recall showed a negative correlation with mean diffusivity, radial diffusivity, and axial diffusivity of the bilateral inferior longitudinal fasciculus, right uncinate fasciculus, and bilateral cerebellum (posterior lobe), whereas delayed recall showed a negative correlation with the right anterior thalamic radiations, right inferior longitudinal fasciculus, and right uncinate fasciculus.

7) Visual memory immediate recall showed a negative correlation with mean diffusivity, radial diffusivity, and axial diffusivity of the right inferior longitudinal fasciculus, whereas delayed recall negatively correlated with diffusivity measurements of the right anterior thalamic radiations and bilateral inferior longitudinal fasciculus.

There was no statistically significant correlation between fractional anisotropy and any of the neuropsychology assessment scores in the ETCI group. No significant correlation was found between neuropsychological test scores and any of the diffusion measures in the ETNCI and HC groups.

\section{DISCUSSION}

Cognitive impairments often accompany typical motor symptoms of ET. Cognitive impairments related to impairments in the frontal-cerebellar circuit appear to be the most prominent feature of ET. ${ }^{4}$ Despite the high prevalence of ET and the impact of cognitive impairments on quality of life, the spectrum and neural basis of these impairments are not fully characterized. Although a number of DTI studies have been conducted to better understand ET pathology, none have focused on the cognitive correlates of the disease. To our knowledge, our study is the first to comprehensively study the broad range of cognitive deficits in ET by using a structured neuropsychological battery and to correlate them with the structural abnormalities identified by using DTI.

We analyzed DTI measures that are thought to reflect the following: 1) directional WM integrity (fractional anisotropy), 2) tissue breakdown with increased water content (mean diffusivity), and 3) the integrity of axons versus their adjacent myelin sheaths (radial diffusivity and axial diffusivity). ${ }^{20}$ Diffusion anisotropy (fractional anisotropy) may not be enough to better characterize the tissue microstructure. If diffusion changes along the direction of the semi-major axis (axial diffusivity) are proportional to those of the semi-minor axes (radial diffusivity), then fractional anisotropy, which is the ratio of axial diffusivity and radial diffusivity, would remain relatively unchanged. ${ }^{21}$ Thus, it is recommended that studies use multiple diffusion tensor measures 
to assess WM integrity comprehensively. ${ }^{21}$ Our analysis revealed changes in the ETCI group mainly involving the bilateral frontal parietal WM. The changes were mainly observed in mean diffusivity, radial diffusivity, and axial diffusivity. This finding is similar to those in studies performing whole-brain analysis by using TBSS in patients with ET. ${ }^{9,10}$ Significant changes in fractional anisotropy of the cerebrum and cerebellum have been reported in various region-of-interest-based studies in patients with ET. ${ }^{8,9}$ The reason for these changes is unclear, but a possible explanation might be attributable to subtle changes in fractional anisotropy that can be detected within ROIs, which however may not survive stringent whole-brain statistical analysis. A region-of-interest approach can be artifactual because even a subtle amount of atrophy in a tract of interest could lead to wrong placement of ROIs. ${ }^{21}$ Thus, region-of-interest results need to be interpreted with caution.

Executive function is often regarded as the most common cognitive deficit in patients with ET. ${ }^{4}$ In the present study, executive and working memory dysfunction showed a correlation with DTI measures in the right medial frontal gyrus, right cingulum, bilateral anterior thalamic radiations, bilateral superior longitudinal fasciculus, left uncinate fasciculus, and bilateral cerebellum (posterior lobe). Our results revealed that executive dysfunction in ET was largely associated with changes in frontal WM. Lesion studies have demonstrated that the frontal lobe is crucial for executive functions. ${ }^{22}$ Zheng et $\mathrm{al}^{23}$ also reported involvement of frontal connections while testing DTI correlates of executive dysfunction in Parkinson disease. However, a meta-analysis of neuroimaging and lesion studies revealed that executive functions are sensitive, but not specific, to frontal lobe functioning, meaning that both frontal and nonfrontal brain regions are necessary for executive functions. $^{24}$

Our results are in agreement with this finding. We also found correlation of executive dysfunction with temporal lobe projections. The temporal lobe has several WM projections, including the cingulum, which are involved in many brain functions, such as working memory and attention. ${ }^{25}$ The superior longitudinal fasciculus, inferior longitudinal fasciculus, and uncinate fasciculus have an important role in executive function, language, and memory. ${ }^{26}$ Our results are suggestive of a possible relationship between WM integrity in the anterior thalamic radiations and executive performance. This finding is consistent with the role of the anterior thalamic radiations in cognition because the anterior thalamic radiations carry fibers from the thalamic nuclei to the prefrontal cortex, which are involved in executive function and planning complex behaviors. ${ }^{27-30}$ Thalamic atrophy has been related to cognitive performance in many neurodegenerative disorders, such as Huntington disease, mostly affecting the executive functioning of patients. ${ }^{31}$ Thus, our finding of a correlation between anterior thalamic radiations-impaired diffusivity and executive dysfunction is consistent with prior work, suggesting the importance of thalamic-prefrontal connections in executive control.

Neuropathologic research supports the role of cerebellar degeneration in the pathogenesis of ET, but its role in cognitive dysfunction remains unclear. ${ }^{32}$ Research in patients with focal lesions (strokes or tumors) and fMRI in healthy subjects has shown the role of the cerebellum in a variety of cognitive functions. ${ }^{11}$ An fMRI study by Passamonti et al ${ }^{33}$ showed abnormal activation of the cingulum and cerebellum (posterior lobe) in patients with ET during working memory trials. In our previous study on the correlation of GM volume with cognitive dysfunction in ET, we found that executive dysfunction correlated with GM volume values in the frontal lobe, cingulum, and cerebellum (posterior lobe). ${ }^{34}$ The overactivation of the cingulum and cerebellum during working memory trials in an fMRI study ${ }^{33}$ may represent compensation for volume loss and loss of WM integrity of these areas observed in our study.

We observed significant impairment of the visuospatial functions in ETCI, an observation similar to that reported by Sahin et al. ${ }^{3}$ Daniels et $\mathrm{al}^{35}$ reported relative expansion of GM volume bilaterally in the region of the temporal parietal junction in patients with ET and attributed this to adaptive reorganization for compromised visuospatial functioning. In the present study, visuospatial dysfunction was correlated with diffusion measures of the right parieto-occipital lobe. Our previous study also found a correlation of visuospatial dysfunction with GM volume values in the parietal, temporal, and occipital lobes. ${ }^{34}$ The right occipital and right parietal cortices are specialized for visuospatial function. ${ }^{36}$ The correlation of the visuospatial dysfunction score with GM volume changes and diffusion parameters in these areas may suggest that structural damage in these areas may contribute to underlying dysfunction.

In the present study, we also found impairment in visual and verbal memory correlating with diffusion measures of the right anterior thalamic radiations, bilateral inferior longitudinal fasciculus, right uncinate fasciculus, and posterior lobe of the cerebellum. The inferior longitudinal fasciculus is known to play an important role in visual memory as demonstrated by previous postmortem, lesion, and imaging studies. ${ }^{26}$ The uncinate fasciculus plays a role in the formation and retrieval of memories. ${ }^{26}$ Thus, memory impairment seen in patients with ET may represent involvement of the cerebellothalamocortical loop. The involvement of memory seen in our study may help explain the risk of dementia in patients with ET, as demonstrated by a few previous studies. ${ }^{37,38}$

In our study, an association was observed between cognition and WM microstructure in ET. Such an association has been demonstrated in different degenerative disorders such as Parkinson disease and Alzheimer disease. ${ }^{23,39}$ It suggests a role of neurodegeneration in cognitive deficits seen in ET. Neuropathologic studies also provide evidence of neurodegeneration in ET. ${ }^{32}$ Novellino et al, ${ }^{40}$ for the first time, demonstrated increased iron accumulation in a group of patients with ET by using T2* MR imaging, further strengthening the role of neurodegeneration in ET. Prospective longitudinal neuroimaging studies coupled with neuropathology are needed to substantiate the role of neurodegeneration in ET.

\section{Limitations}

Even though careful clinical examination was performed to exclude Parkinson disease, a dopamine transporter scan to demonstrate the integrity of the nigrostriatal network was not performed. TBSS definitely has its advantages in DTI analysis; 
nonetheless, it is not without limitations. In regions of crossing tracts or junctions, it may be inefficient in detecting changes in DTI parameters. Finally, the results of our correlation analysis should be interpreted carefully because they were not controlled for multiple comparisons, which may lead to false-positives. Moreover, in view of the cross-sectional design of the present study, future longitudinal studies would be needed to better assess the course of neuroanatomic changes and cognitive impairment in ET.

\section{CONCLUSIONS}

This study demonstrates microstructural changes in the WM of patients with ET with cognitive impairment. These changes correlated with abnormal neuropsychological test scores in various brain regions. Such mapping of WM networks may provide insight into the etiology of cognitive impairment in ET.

\section{REFERENCES}

1. Lombardi WJ, Woolston DJ, Roberts JW, et al. Cognitive deficits in patients with essential tremor. Neurology 2001;57:785-90

2. Chandran V, Pal PK, Reddy JY, et al. Non-motor features in essential tremor. Acta Neurol Scand 2012;125:332-37

3. Sahin HA, Terzi M, Ucak S, et al. Frontal functions in young patients with essential tremor: a case comparison study. J Neuropsychiatry Clin Neurosci 2006;18:64-72

4. Gasparini M, Bonifati V, Fabrizio E, et al. Frontal lobe dysfunction in essential tremor: a preliminary study. J Neurol 2001;248:399-402

5. Bagepally BS, Bhatt MD, Chandran V, et al. Decrease in cerebral and cerebellar gray matter in essential tremor: a voxel-based morphometric analysis under 3T MRI. J Neuroimaging 2012;22:275-78

6. Benito-León J, Alvarez-Linera J, Hernandez-Tamames JA, et al. Brain structural changes in essential tremor: voxel-based morphometry at 3-Tesla. J Neurol Sci 2009;287:138-42

7. Martinelli P, Rizzo G, Manners D, et al. Diffusion-weighted imaging study of patients with essential tremor. Mov Disord 2007;22: 1182-85

8. Shin DH, Han BS, Kim HS, et al. Diffusion tensor imaging in patients with essential tremor. AJNR Am J Neuroradiol 2008;29:151-53

9. Klein JC, Lorenz B, Kang JS, et al. Diffusion tensor imaging of white matter involvement in essential tremor. Hum Brain Mapp 2011;32:896-904

10. Saini J, Bagepally BS, Bhatt MD, et al. Diffusion tensor imaging: tract based spatial statistics study in essential tremor. Parkinsonism Relat Disord 2012;18:477-82

11. Schmahmann JD. From movement to thought: anatomic substrates of the cerebellar contribution to cognitive processing. Hum Brain Mapp 1996;4:174-98

12. Chouinard S, Louis ED, Fahn S. Agreement among movement disorder specialists on the clinical diagnosis of essential tremor. Mov Disord 1997;12:973-76

13. Petersen RC, Doody R, Kurz A, et al. Current concepts in mild cognitive impairment. Arch Neurol 2001;58:1985-92

14. Moering RG, Schinka JA, Mortimer JA, et al. Normative data for elderly African Americans for the Stroop Color and Word Test. Arch Clin Neuropsychol 2004;19:61-71

15. Benton AL, Varney NR, Hamsher KD. Visuospatial judgment: a clinical test. Arch Neurol 1978;35:364-67

16. Smith SM, Jenkinson M, Johansen-Berg H, et al. Tract-based spatial statistics: voxelwise analysis of multi-subject diffusion data. Neuroimage 2006;31:1487-505

17. Behrens TE, Woolrich MW, Jenkinson M, et al. Characterization and propagation of uncertainty in diffusion-weighted MR imaging. Magn Reson Med 2003;50:1077-88

18. Smith SM, Jenkinson M, Woolrich MW, et al. Advances in functional and structural MR image analysis and implementation as FSL. Neuroimage 2004;23(suppl 1):S208-19

19. Smith SM, Johansen-Berg H, Jenkinson M, et al. Acquisition and voxelwise analysis of multi-subject diffusion data with tract-based spatial statistics. Nat Protoc 2007;2:499-503

20. Oertel-Knöchel V, Reinke B, Alves G, et al. Frontal white matter alterations are associated with executive cognitive function in euthymic bipolar patients. J Affect Disord 2014;155:223-33

21. Acosta-Cabronero J, Williams GB, Pengas G, et al. Absolute diffusivities define the landscape of white matter degeneration in Alzheimer's disease. Brain 2010;133:529-39

22. Rowe AD, Bullock PR, Polkey CE, et al. "Theory of mind" impairments and their relationship to executive functioning following frontal lobe excisions. Brain 2001;124:600-16

23. Zheng Z, Shemmassian S, Wijekoon C, et al. DTI correlates of distinct cognitive impairments in Parkinson's disease. Hum Brain Mapp 2014;35:1325-33

24. Alvarez JA, Emory E. Executive function and the frontal lobes: a meta-analytic review. Neuropsychol Rev 2006;16:17-42

25. Sepulcre J, Masdeu JC, Pastor MA, et al. Brain pathways of verbal working memory: a lesion-function correlation study. Neuroimage 2009;47:773-78

26. Sasson E, Doniger GM, Pasternak O, et al. White matter correlates of cognitive domains in normal aging with diffusion tensor imaging. Front Neurosci 2013;7:32

27. Floresco SB, Grace AA. Gating of hippocampal-evoked activity in prefrontal cortical neurons by inputs from the mediodorsal thalamus and ventral tegmental area. J Neurosci 2003;23:3930-43

28. Mamah D, Conturo TE, Harms MP, et al. Anterior thalamic radiation integrity in schizophrenia: a diffusion-tensor imaging study. Psychiatry Res 2010;183:144-50

29. Van der Werf YD, Jolles J, Witter MP, et al. Contributions of thalamic nuclei to declarative memory functioning. Cortex 2003;39: 1047-62

30. Zoppelt D, Koch B, Schwarz M, et al. Involvement of the mediodorsal thalamic nucleus in mediating recollection and familiarity. $\mathrm{Neu}$ ropsychologia 2003;41:1160-70

31. Kassubek J, Juengling FD, Ecker D, et al. Thalamic atrophy in Huntington's disease co-varies with cognitive performance: a morphometric MRI analysis. Cereb Cortex 2005;15:846-53

32. Louis ED, Faust PL, Vonsattel JP, et al. Neuropathological changes in essential tremor: 33 cases compared with 21 controls. Brain 2007;130:3297-307

33. Passamonti L, Novellino F, Cerasa A, et al. Altered cortical-cerebellar circuits during verbal working memory in essential tremor. Brain 2011;134:2274-86

34. Bhalsing KS, Upadhyay N, Kumar KJ, et al. Association between cortical volume loss and cognitive impairments in essential tremor. Eur J Neurol 2014;21:874-83

35. Daniels C, Peller M, Wolff S, et al. Voxel-based morphometry shows no decreases in cerebellar gray matter volume in essential tremor. Neurology 2006;67:1452-56

36. Nobre AC, Sebestyen GN, Gitelman DR, et al. Functional localization of the system for visuospatial attention using positron emission tomography. Brain 1997;120(pt 3):515-33

37. Benito-León J, Louis ED, Bermejo-Pareja F. Elderly-onset essential tremor is associated with dementia. Neurology 2006;66:1500-05

38. Bermejo-Pareja F, Louis ED, Benito-Leon J. Risk of incident dementia in essential tremor: a population-based study. Mov Disord 2007;22:1573-80

39. Fellgiebel A, Schermuly I, Gerhard A, et al. Functional relevant loss of long association fibre tracts integrity in early Alzheimer's disease. Neuropsychologia 2008;46:1698-706

40. Novellino F, Cherubini A, Chiriaco C, et al. Brain iron deposition in essential tremor: a quantitative 3-Tesla magnetic resonance imaging study. Mov Disord 2013;28:196-200 